\title{
Detection of thermal radio emission from a single coronal giant
}

\author{
E. O'Gorman ${ }^{1}$, G. M. Harper ${ }^{2}$, and W. Vlemmings ${ }^{3}$
}

\author{
1 Dublin Institute for Advanced Studies, 31 Fitzwilliam Place, Dublin 2, Ireland \\ e-mail: ogorman@cp.dias.ie \\ 2 Center for Astrophysics and Space Astronomy, University of Colorado, 389 UCB, Boulder, CO 80309, USA \\ 3 Department of Earth and Space Sciences, Chalmers University of Technology, Onsala Space Observatory, 43992 Onsala, Sweden
}

Received 18 August 2016 / Accepted 22 November 2016

\begin{abstract}
We report the detection of thermal continuum radio emission from the K0 III coronal giant Pollux ( $\beta$ Gem) with the Karl G. Jansky Very Large Array (VLA). The star was detected at 21 and $9 \mathrm{GHz}$ with flux density values of $150 \pm 21$ and $43 \pm 8 \mu \mathrm{Jy}$, respectively. We also place a $3 \sigma_{\text {rms }}$ upper limit of $23 \mu \mathrm{Jy}$ for the flux density at $3 \mathrm{GHz}$. We find the stellar disk-averaged brightness temperatures to be approximately 9500,15000 , and $<71000 \mathrm{~K}$, at 21,9 , and $3 \mathrm{GHz}$, respectively, which are consistent with the values of the quiet Sun. The emission is most likely dominated by optically thick thermal emission from an upper chromosphere at 21 and $9 \mathrm{GHz}$. We discuss other possible additional sources of emission at all frequencies and show that there may also be a small contribution from gyroresonance emission above active regions, coronal free-free emission and free-free emission from an optically thin stellar wind, particularly at the lower frequencies. We constrain the maximum mass-loss rate from Pollux to be less than $3.7 \times 10^{-11} M_{\odot} \mathrm{yr}^{-1}$ (assuming a wind terminal velocity of $215 \mathrm{~km} \mathrm{~s}^{-1}$ ), which is about an order of magnitude smaller than previous constraints for coronal giants and is in agreement with existing predictions for the mass-loss rate of Pollux. These are the first detections of thermal radio emission from a single (i.e., non-binary) coronal giant and demonstrate that low activity coronal giants like Pollux have atmospheres at radio frequencies akin to the quiet Sun.
\end{abstract}

Key words. stars: atmospheres - radio continuum: stars - stars: chromospheres - stars: coronae - stars: evolution - stars: mass-loss

\section{Introduction}

Unlike low mass stars, moderate-mass $\left(M_{\star} \sim 3 M_{\odot}\right)$ late $\mathrm{B}$ and early A spectral type stars lose little angular momentum during their main-sequence (MS) phase (Wolff \& Simon 1997). Once shell hydrogen burning commences, these stars move away from the MS and begin to form surface convection zones while evolving rapidly through the $F$ and $G$ spectral types. As the surface convection zone deepens, coronal activity is activated and the star ascends the red giant branch (RGB). Subsequently it returns close to the base of the RGB where it becomes a "clump" giant burning helium in its core (Ayres et al. 1998). This coronal activity will inevitably subside as the star spins down. Therefore, late B and early A spectral type stars which do not have coronae on the MS, will develop coronae during a period of their post MS evolution. This is in contrast to lower mass stars like the Sun, which display strong coronal emission on the MS due to intense rotation-induced dynamo-driven magnetic activity (Parker 1970), which subsides as they evolve to red giants.

The atmospheres of coronal giants have been extensively studied at both X-ray (Haisch et al. 1991; Maggio et al. 1990; Huensch et al. 1996) and ultra-violet (UV) wavelengths (Ayres et al. 2003; Dupree et al. 2005). X-ray studies have revealed that stellar coronae are common in the giant branch for spectral types earlier than K1 III but become rare redward of K1 III, a region which has thus been dubbed the "coronal graveyard" (Ayres et al. 1991). The UV emission line studies agree with these findings but also suggest pervasive transition region material at $10^{5} \mathrm{~K}$ among many of the non-coronal giants (Ayres et al. 1997).
Detecting radio emission from coronal giants provides an alternative method to study their atmospheres and allows a comparison with the atmospheres of coronal MS stars. Active coronal MS stars can have intense radio luminosities many orders of magnitude greater than that of the disk-averaged quiet (i.e., active region free) solar value. These high values have been associated with gyrosynchrotron emission from a continuously generated population of non-thermal electrons in their coronae (Linsky \& Gary 1983). Less active coronal MS stars, like the Sun, emit only thermal emission at radio frequencies in their quiescent (i.e., non-flaring) states which is entirely due to freefree processes. Subsequently, their radio luminosities are much lower than those from more active coronal MS stars and only recently has this thermal radio emission been detected from MS stars other than the Sun (Villadsen et al. 2014). Unlike coronal MS stars, little is known about the radio atmospheres of coronal giants. Drake \& Linsky (1986) observed a number of nearby coronal giants at $5 \mathrm{GHz}$ (i.e., $6 \mathrm{~cm}$ ) with the Very Large Array. They failed to detect any single coronal giants but did detect the binary Capella ( $\alpha$ Aur; G5 III + F9 III), which is a long period RS CVn type system. The radio emission from this system was spatially unresolved and was interpreted to be thermal free-free emission from one or both of their chromospheres and coronae.

In this paper we report radio observations of the closest coronal giant, Pollux ( $\beta$ Gem; K0 III), whose basic properties are summarized in Table 1. According to Aurière et al. (2009), Pollux is probably a descendent of an early-A spectral type MS star and now lies either at the base of the red giant branch or is a clump giant. Pollux is a weakly-active magnetic giant and a sub-Gauss value has been obtained for its surface averaged 
Table 1. Basic stellar properties of Pollux ( $\beta \mathrm{Gem})$.

\begin{tabular}{|c|c|c|}
\hline Parameter & Value & Reference \\
\hline HD number. & 62509 & - \\
\hline Spectral type . & K0 III & 1 \\
\hline Effective temperature $T_{\text {eff }}$ & $4904 \pm 50 \mathrm{~K}$ & 2 \\
\hline Distance $d \ldots \ldots \ldots . . .$. & $10.36 \pm 0.03 \mathrm{pc}$ & 3 \\
\hline Angular diameter $\phi_{\star}$ & $7.95 \pm 0.09$ mas & 4 \\
\hline Mass $M_{\star} \ldots \ldots \ldots$ & $1.91 \pm 0.09 M_{\odot}$ & 5 \\
\hline Radius $R_{\star}$ & $8.85 \pm 0.10 R_{\odot}$ & c \\
\hline$[\mathrm{Fe} / \mathrm{H}]$. & 0.08 & 1 \\
\hline Escape velocity $v_{\downarrow}^{\text {esc }}$ & $287 \mathrm{~km} \mathrm{~s}^{-1}$ & $\mathrm{c}$ \\
\hline Age $\ldots \ldots \ldots \ldots \ldots \ldots$ & $1.2 \pm 0.3 \mathrm{Gyr}$ & 5 \\
\hline Soft X-ray luminosity $L_{\mathrm{x}}$ & $10^{27.13} \mathrm{erg} \mathrm{s}^{-1}$ & 7 \\
\hline
\end{tabular}

References. (1) Gray et al. (2003); (2) Takeda et al. (2008); (3) van Leeuwen (2007); (4) Nordgren et al. (2001); (5) Hatzes et al. (2012); (6) Gray (2014); (7) Sanz-Forcada et al. (2011). "c" indicates values calculated in this work.

longitudinal magnetic field (Aurière et al. 2009). It has an X-ray luminosity that is 3 orders of magnitude lower than Capella's (Huensch et al. 1996), despite its similar size to both of the stars in the Capella binary. Extensive studies of Pollux's radial velocity variations have revealed it to have a planetary companion with a period of 560 days and a minimum mass of $2.9 M_{\text {Jup }}$ (Reffert et al. 2006; Hatzes et al. 2006). Understanding the radio properties of the stellar host of an exoplanet not only provides insight on the host itself but also provides insight on the feasibility of detecing radio emission from the exoplanet itself.

\section{Observations and data reduction}

Pollux was observed with the Karl G. Jansky Very Large Array (VLA) in A configuration (Program code: 12B-108, PI: Laurent Chemin) during 2012 October at $K$ band $(18.0-26.5 \mathrm{GHz})$ and again during 2013 January at both $X$ band $(8.0-12.0 \mathrm{GHz})$ and $S$ band $(2.0-4.0 \mathrm{GHz})$. The original goal of this project was to test whether the signature of Pollux's exoplanet could be detected in its orbital reflex motion with a future astrometric Very Long Baseline Array (VLBA) program. The low levels of radio flux density that we report in this paper would appear to rule out such a possibility with current facilities. A brief overview of these observations is provided in Table 2 . The receivers recorded continuum emission with 8-bit samplers in $2 \mathrm{GHz}$ wide windows centered at 21.2 ( $K$ band), 9.0 ( $X$ band), and $3.0 \mathrm{GHz}$ ( $S$ band). For all frequency setups, 3C286 was used as the absolute flux density and bandpass calibrator, while J0741+3112 was used as the complex gain calibrator. Pollux was observed offset $\sim 8^{\prime \prime}$ due west of the phase reference center and so any potential interferometric artefacts at phase center could not be mistaken for the source.

Flagging and calibration were performed with the Common Astronomy Software Applications (CASA) package (McMullin et al. 2007) version 4.2.2 using the VLA calibration pipeline version 1.3.1 and some manual flagging. Images were created using the CLEAN algorithm (Högbom 1974) in full Stokes, with Briggs weighting and a robust parameter of 0.5 . At $S$ band, we were required to image a number of bright nearby (i.e., a few arcminutes) serendipitous sources to suppress their sidelobe contamination throughout the image. In the final primary beam-corrected images, the rms noise per synthesized beam close to the source was $14 \mu \mathrm{Jy} \mathrm{beam}^{-1}$ at $K$ band $(21.2 \mathrm{GHz}), 6 \mu \mathrm{Jy}$ beam $^{-1}$ at $X$ band $(9.0 \mathrm{GHz})$, and
$7.5 \mu \mathrm{Jy}$ beam $^{-1}$ at $S$ band $(3.0 \mathrm{GHz})$. The source flux density and position were determined by fitting a point source to the calibrated visibilities using the CASA task uvmodelfit. To mitigate the possibility of decorrelation of the visibilities at $21.2 \mathrm{GHz}$, which could result in flux scale errors, we limited the point source fit to baselines less than $500 \mathrm{k} \lambda$ at this frequency. The flux density uncertainty for standard VLA observations is $\sim 3 \%$ at $S$ and $X$ band, and $\sim 10 \%$ at $K$ band. The error bars throughout this paper do not include this uncertainty in the absolute flux calibration but reflect both the statistical root-mean-square (rms) error values, $\sigma_{\mathrm{s}}$, obtained from the final primary beam corrected images, and the formal fitting uncertainty, $\sigma_{\mathrm{f}}$. The total error in the flux density is then assumed to be, $\sigma_{\mathrm{t}}=\left(\sigma_{\mathrm{s}}^{2}+\sigma_{\mathrm{f}}^{2}\right)^{0.5}$.

\section{Results}

Pollux was detected (i.e., at $>3 \sigma_{\text {rms }}$ significance) at 21.2 and $9 \mathrm{GHz}$ in Stokes $I$ (total intensity) in both the point source fits to the calibrated visibilities and in the corresponding radio images, which are shown in Fig. 1. The detections had signal-to-noise ratios $(\mathrm{S} / \mathrm{N})$ of 7 at $21.2 \mathrm{GHz}$ and 5 at $9.0 \mathrm{GHz}$. The star was not detected at $3 \mathrm{GHz}$ or in any other Stokes parameter. The derived flux densities along with the $3 \mathrm{GHz} 3 \sigma_{\text {rms }}$ upper limit are listed in Table 2 and are consistent with the previous non-detection of Pollux by Drake \& Linsky (1986) who reported a $3 \sigma_{\text {rms }}$ upper limit of $340 \mu \mathrm{Jy}$ at $5 \mathrm{GHz}$. We fitted an elliptical Gaussian to the source using CASA's imfit task to confirm that the star was unresolved at both 21.2 and $9.0 \mathrm{GHz}$.

The expected positions of Pollux at the two observational epochs were calculated using the HIPPARCOS coordinates, proper motion, and parallax (van Leeuwen 2007). The positional offset of the source could then be determined from the difference between the expected position and the actual measured position. At both 21.2 and $9 \mathrm{GHz}$, we find that the positional offset is smaller than the noise-based uncertainty, which we define as the synthesized beam HPBW divided by $2 \times S / N$ of the source (Condon et al. 1998). This good agreement confirms that we have detected Pollux at 21.2 and $9 \mathrm{GHz}$. In Fig. 1 it can be seen that a $3 \sigma_{\text {rms }}$ peak lies close to the expected position of Pollux at $3 \mathrm{GHz}$. However, considering that this peak lies at a distance of almost two times the noise-based uncertainty away from the expected position, along with the fact that there are other peaks of comparable statistical significance close by, we do not attempt to consider this peak as a detection.

\section{Discussion}

\subsection{Spectral indices and brightness temperatures}

Figure 2 shows the radio spectral energy distribution for Pollux. We find that the spectral index $\alpha$ (where $S_{v} \propto v^{\alpha}$ ) has a value of $\alpha=1.5 \pm 0.4$ between 9 and $21.2 \mathrm{GHz}$, and has a value of $\alpha>0.6 \pm 0.5$ between 3 and $9 \mathrm{GHz}$. These spectral index values are in broad agreement with the spectral indices of the disk-averaged quiet Sun over the same frequency ranges, i.e., $\alpha_{\odot}=1.75$ between 6 and $400 \mathrm{GHz}$, and $\alpha_{\odot}=0.56$ between 0.35 and $6 \mathrm{GHz}$ (Benz 2009). It can be seen that the flux densities at 9 and $21.2 \mathrm{GHz}$ are significantly above the values expected from a blackbody with a photospheric angular diameter and a temperature equal to the effective temperature. The derived brightness temperatures are listed in Table 2 and are consistent with the solar minimum values. 
Table 2. VLA observations of Pollux.

\begin{tabular}{cccccccc}
\hline \hline Date & Band & $\begin{array}{c}\text { Center } \\
\text { frequency } \\
(\mathrm{GHz})\end{array}$ & $\begin{array}{c}\text { Bandwidth } \\
(\mathrm{GHz})\end{array}$ & $\begin{array}{c}\text { Time on } \\
\text { source } \\
(\mathrm{min})\end{array}$ & $\begin{array}{c}\text { Restoring } \\
\text { beam HPBW } \\
\left({ }^{\prime \prime} \times{ }^{\prime \prime}\right)\end{array}$ & $\begin{array}{c}\text { Flux } \\
\text { density } \\
(\mu \mathrm{Jy})\end{array}$ & $\begin{array}{c}\text { Brightness } \\
\text { temperature } \\
(\mathrm{K})\end{array}$ \\
\hline 2012 Oct. 14 & $K$ & 21.2 & 2.0 & 32 & $0.092 \times 0.085$ & $150 \pm 21$ & $9300 \pm 1300$ \\
2013 Jan. 01 & $X$ & 9.0 & 2.0 & 17 & $0.381 \times 0.218$ & $43 \pm 8$ & $14800 \pm 2800$ \\
2013 Jan. 01 & $S$ & 3.0 & 2.0 & 17 & $1.194 \times 0.534$ & $<23$ & $<71000$ \\
\hline
\end{tabular}

Notes. The brightness temperatures are calculated from Eq. (1), where we have assumed that the stellar diameter is equal to the optical diameter and that the star is a uniform disk.
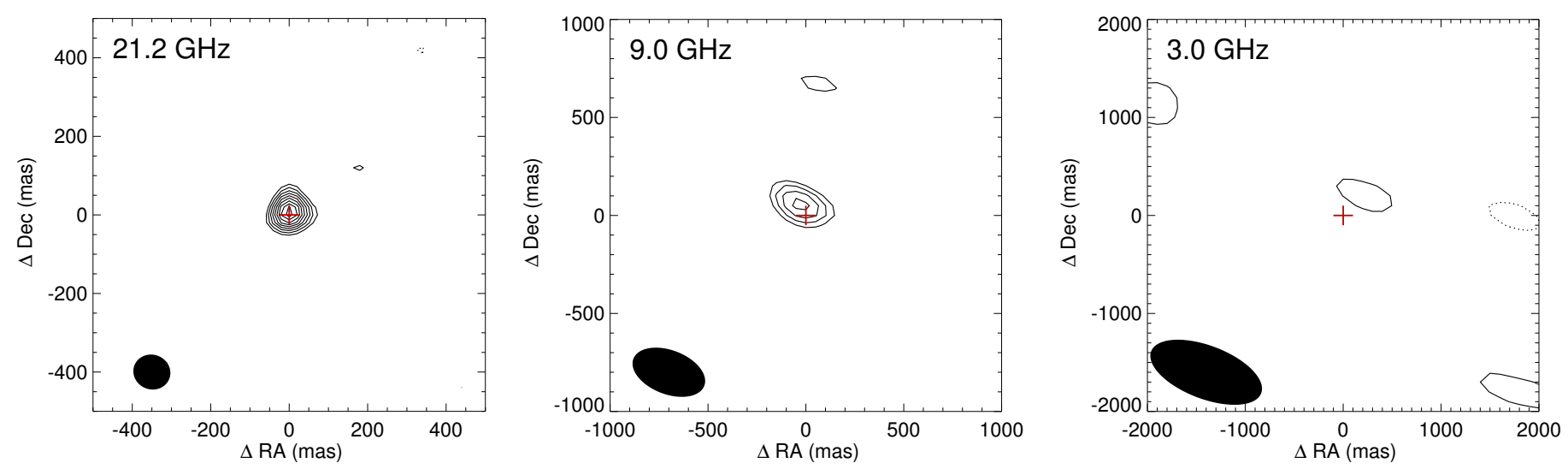

Fig. 1. Radio images of Pollux at $21.2 \mathrm{GHz}$ (left), $9 \mathrm{GHz}$ (middle), and $3 \mathrm{GHz}$ (right). Contours are set to $(-3,3,4, \ldots 11) \times \sigma_{\mathrm{rms}}$ where $\sigma_{\mathrm{rms}}$ is the rms noise in each image. The red cross marks the expected position of the photosphere at the epoch of each observation. The restoring beam is shown in the bottom left of each image. Note the scales on the axes in each panel double in size from left to right.

\subsection{Radio emission mechanisms}

In the following sections we discuss the possible radio emission mechanisms responsible for the observed radio flux from Pollux. We note that our discussion naturally follows the discussions outlines already presented in Drake et al. (1993) and Villadsen et al. (2014). In Drake et al. (1993), the possible radio emission mechanisms responsible for their $8.3 \mathrm{GHz}$ detections of the subgiant star Procyon are discussed. A similar layout is applied in Villadsen et al. (2014) to discuss their $34.5 \mathrm{GHz}$ detections of three solar-type MS stars (i.e., $\tau$ Cet, $\eta$ Cas A, and 40 Eri A).

\subsubsection{Free-free emission from a chromosphere and transition region}

If Pollux were a perfect blackbody with angular diameter $\phi=\phi_{\star}$ and brightness temperature $T_{\mathrm{b}}=T_{\text {eff }}$ at any radio frequency $v$, then the observed flux density would be

$S_{v}=0.18 \mu \mathrm{Jy}\left(\frac{T_{\mathrm{b}}}{4904 \mathrm{~K}}\right)\left(\frac{v}{\mathrm{GHz}}\right)^{2}\left(\frac{\phi}{7.95 \mathrm{mas}}\right)^{2}$.

This blackbody radio spectrum is plotted in Fig. 2 along with our flux density measurements which are clearly all in excess of this spectrum. This is because Pollux, like the Sun, has a chromosphere and a transition region. At radio frequencies, electrons and ions from these regions are a source of free-free opacity which increases as frequency decreases. This means that at lower radio frequencies an optical depth of unity is reached higher in the atmosphere where the gas/electron temperature $T_{\mathrm{e}}>T_{\text {eff }}$ and $\phi>\phi_{\star}$, and so the flux density will be greater than that expected from a blackbody alone ${ }^{1}$ (e.g., Cassinelli \& Hartmann 1977). In Fig. 2 we also include the predicted radio spectral energy distribution from the atmospheric model of Pollux by Sim (2001). This model contains a turbulently extended chromosphere, a transition region and corona and is in reasonably agreement with our measurements. We note that the semi-empirical model is not a close match to the observed H I Lyman profiles, and therefore the model itself has intrinsic uncertainties. The radio emission from this model atmosphere is expected to be in excess of the blackbody emission and this excess is expected to be greater at lower frequencies, in agreement with our measurements.

In Fig. 3 we compare the disk-averaged brightness temperatures of Pollux against the solar minimum and maximum values taken from White (2004). The brightness temperature of the Sun changes between solar minimum and maximum mainly because of gyroresonance emission above active regions which will be discussed further in Sect. 4.2.3. At solar minimum, radio emission between 3 and $21 \mathrm{GHz}$ is dominated by optically thick thermal emission from from the upper chromosphere and lower transition region. The relationship between the radio optical depths in the quiet Sun and Pollux can be estimated from the chromospheric scaling relations of Ayres (1979). The chromospheric free-free optical depth scales as $\tau \propto n_{\mathrm{e}}^{2} H$, where $n_{\mathrm{e}}$ is the electron density and $H$ is the density scale-height. $H$ scales as $\propto T_{\text {eff }} / g_{*}$, where $g_{*}$ is the surface gravity, while $\left\langle n_{\mathrm{e}}\right\rangle \propto \sqrt{g_{*}} T_{\text {eff }}^{5 / 2}$, so that the surface gravity terms cancel (Harper et al. 2013). The remaining $T_{\text {eff }}^{6}$ term gives $\tau_{\text {Pollux }} / \tau_{\odot} \sim 0.4$ which suggests that Pollux will have a slightly lower opacity chromosphere, and for a given

\footnotetext{
1 At very high frequencies (i.e., $v \gtrsim 1 \mathrm{THz}$ ) the temperature minimum will be probed, in which case $T_{\mathrm{b}}<T_{\text {eff }}$ and the flux density will be less than that expected from a blackbody (e.g., Liseau et al. 2013).
} 


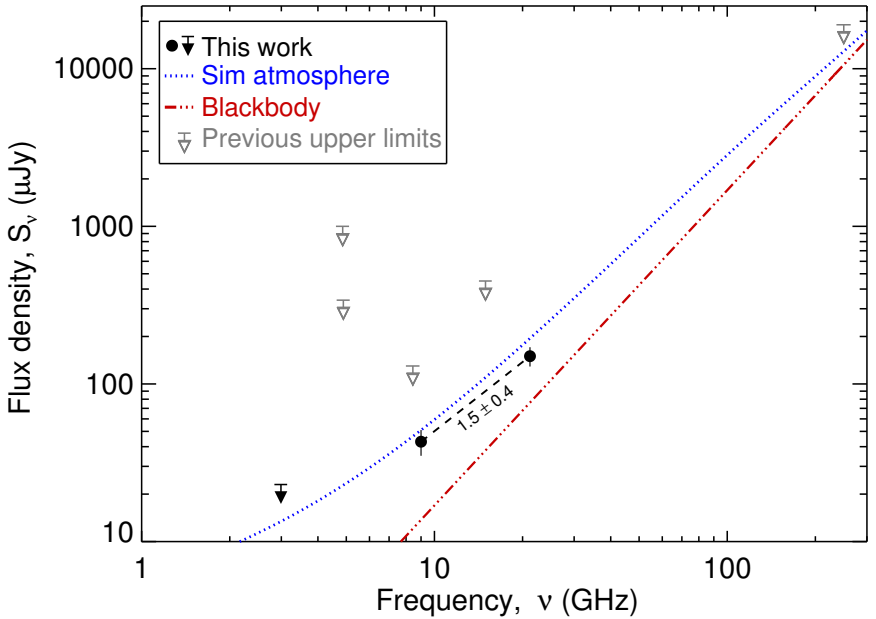

Fig. 2. Radio spectral energy distribution for Pollux between 1 and $300 \mathrm{GHz}$. The red dash-dotted line is the expected blackbody emission assuming a uniform intensity disk with angular diameter $\phi=\phi_{\star}$ and brightness temperature $T_{\mathrm{b}}=T_{\text {eff. }}$. The black filled circles are the VLA detections at 21.2 and $9 \mathrm{GHz}$. The blue dotted line is the expected spectral energy distribution of Pollux using the model atmosphere from Sim (2001). Our $3 \mathrm{GHz}$ upper limit is also shown along with previous upper limits taken from Wendker (1995). The black dashed line represents a least-square fit to our detections assuming that $S_{v} \propto v^{\alpha}$.

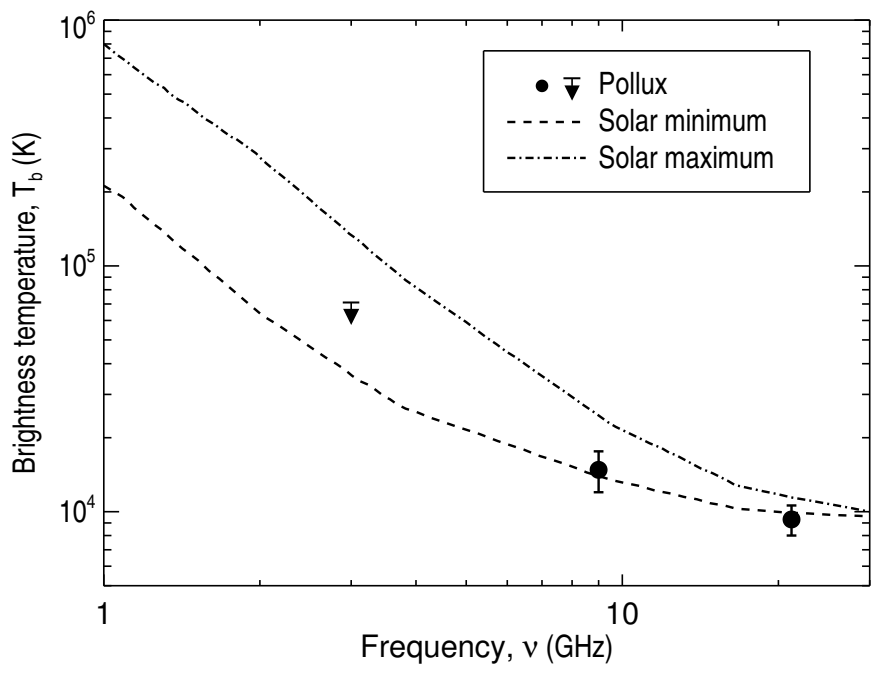

Fig. 3. Derived disk averaged brightness temperature of Pollux at 21.2 and $9 \mathrm{GHz}$, along with the upper limit value at $3 \mathrm{GHz}$. The dashed line is the solar minimum disk averaged brightness temperature while the dash dotted line is the solar maximum disk averaged brightness temperature (White 2004).

frequency, deeper and cooler plasma is probed. Given the similarities between the solar brightness temperatures and the values we find for Pollux, it seems likely that Pollux's radio emission also originates mainly from an upper chromospheric and lower transition region at our observing frequencies.

\subsubsection{Free-free thermal emission from a bound corona}

We now examine the possibility that some of the radio emission results from free-free thermal emission from a bound (i.e., static) optically thin corona. We can immediately rule out an optically thick corona on the basis that the expected brightness temperatures would be much larger than those we find. Since the solar corona is optically thin above a few $\mathrm{GHz}$, the rising spectrum (or positive spectral indices) we deduce for Pollux immediately suggests emission at 9 and $21.2 \mathrm{GHz}$ is not dominated by optically thin coronal emission, because in this case the spectrum would be almost flat (i.e., $S_{v} \propto v^{-0.1}$ ).

Pollux is a weak soft X-ray source (Maggio et al. 1990) and its X-ray $(5-100 \AA)$ luminosity of $10^{27.13} \mathrm{erg} \mathrm{s}^{-1}$ (Sanz-Forcada et al. 2011) is similar to the solar minimum value defined in Judge et al. (2003). Thus, Pollux - with a surface area 78 times that of the Sun - has an X-ray surface flux that is about $1 \%$ of the solar value. This soft X-ray flux originates as thermal emission from an optically thin corona and is the sum of many different thermal processes such as free-free, bound-free, and bound-bound. Knowledge of the coronal emission measure ( $E M$, measured in $\mathrm{cm}^{-3}$ ) allows the expected radio luminosity to be calculated using

$L_{v}=\epsilon_{v}\left(T_{\mathrm{e}}\right) E M$

where $\epsilon_{v}\left(T_{\mathrm{e}}\right)$ is the radiative loss rate of the plasma (in erg s $\mathrm{s}^{-1} \mathrm{~cm}^{3} \mathrm{~Hz}^{-1}$ ) and is assumed to be entirely from free-free interactions. This is calculated using Eqs. (3.54) and (3.55) from Spitzer (1978) and assuming helium to be fully ionized. Using the larger $E M$ value and the corresponding coronal temperature of $T_{\mathrm{e}}=10^{6.23} \mathrm{~K}$ from the two temperature $E M$ fit given in Sanz-Forcada et al. (2011), we find the expected flux density contribution from Pollux's optically thin corona to be $S_{v} \sim 0.5 \mu \mathrm{Jy}$ at $3 \mathrm{GHz}$ and even smaller at higher frequencies. We conclude that the free-free thermal emission from a bound corona has only a very small contribution to our radio detections.

\subsubsection{Gyroresonance emission from active regions}

The non-flaring Sun displays a clear cycle in its radio flux density. This is due to coronal material above active regions becoming optically thick from the presence of hot dense plasma and strong magnetic fields and results in bright spots of coronal temperatures $\left(\sim 10^{6} \mathrm{~K}\right)$ appearing on a cooler chromospheric temperature $\left(\sim 10^{4} \mathrm{~K}\right)$ disk. As the number of active regions changes over a solar cycle, so too does the radio flux density contribution from these bright spots. The dominant source of opacity in these bright spots is free-free thermal electrons in the corona at low frequencies (i.e., $<2 \mathrm{GHz}$ ) and gyroresonance absorption by thermal electrons in the corona between 3 and $\sim 15 \mathrm{GHz}$ (White \& Kundu 1997; Lee et al. 1998). The gyroresonance mechanism is the non-relativistic limit of the gyrosynchrotron mechanism and typically operates at low harmonics ( $s=2-4)$ of the electron gyrofrequency $v_{\mathrm{B}}$, such that

$v=s v_{\mathrm{B}}=2.8 s B \mathrm{MHz}$

for a given magnetic field strength $B$ (in Gauss).

We can use our VLA data to constrain the covering fraction of these coronal bright spots on Pollux, following the arguments outlined in Drake et al. (1993) and Villadsen et al. (2014). We assume that at any given frequency the disk averaged brightness temperature $T_{\mathrm{b}}$ can be modelled as a uniform disk having either a chromospheric or transition region temperature $T_{\text {disk }}$, that is obscured by coronal bright spots of temperature $T_{\text {cor }}$ having a covering fraction $f_{v}$ such that

$T_{\mathrm{b}}=\left(1-f_{v}\right) T_{\mathrm{disk}}+f_{v} T_{\text {cor }}$.

The covering fraction will decrease as frequency increases since higher frequencies correspond to stronger magnetic field 
strengths. For example, coronal magnetic field strengths of 360, 1070 and $2520 \mathrm{G}$ would be required if gyroresonance emission in the third harmonic contributes to the radio flux densities at 3, 9 , and $21.2 \mathrm{GHz}$, respectively. We again use $T_{\text {cor }}=10^{6.23} \mathrm{~K}$, while for $T_{\text {disk }}$, we assume the disk integrated brightness temperature values of the quiet Sun (White 2004), since these measurements will have the least contribution from gyroresonance emission. We find that the brightness temperatures for Pollux at 9 and $21.2 \mathrm{GHz}$ are similar to solar minimum values which could imply little or no contribution from gyroresonance emission at these frequencies. Our upper limit for the disk integrated brightness temperature of Pollux at $3 \mathrm{GHz}$ is well above the solar minimum value of $\sim 3.5 \times 10^{4} \mathrm{~K}$. We can thus derive a maximum covering fraction of $2 \%$ for coronal bright spots at $3 \mathrm{GHz}$ using Eq. (4). For this calculation we have assumed that like the Sun, Pollux contains localized active regions, an assumption which is supported by the discovery of a large scale magnetic field in the photosphere of Pollux (Aurière et al. 2009).

\subsubsection{Free-free emission from an ionized stellar wind}

The unbound solar corona escapes to the interstellar medium through open magnetic field lines at a mean rate of $2.5 \times$ $10^{-14} M_{\odot} \mathrm{yr}^{-1}$ (Athay 1976), forming the solar wind. In general, the mass-loss rates from giant stars are many orders of magnitude greater than the solar value. However, these mass-loss rates are extremely poorly constrained for coronal giants like Pollux. Drake \& Linsky (1986) were able to place $3 \sigma_{\text {rms }}$ upper limits of $\sim 5 \times 10^{-10} M_{\odot} \mathrm{yr}^{-1}$ using their best constrained non-detections of coronal giants.

We can use the multi-frequency VLA detections to estimate the flux density contribution from Pollux's wind and thereby constrain its mass loss rate. To do so we need an approximation of the stellar wind velocity, $v_{\mathrm{w}}$. Pollux does not show any wind scattered Mg II or Lyman alpha features in its HST/STIS spectra while the C III $977 \AA$ and O IV lines in FUSE spectra presented in Dupree et al. (2005) are consistent with no wind scattering. Therefore Pollux does not have either a cool slow velocity wind (i.e., $v_{\mathrm{w}}=10-50 \mathrm{~km} \mathrm{~s}^{-1}$ ) typical from cool giants or a warm intermediate velocity wind (i.e., $v_{\mathrm{w}}=70-150 \mathrm{~km} \mathrm{~s}^{-1}$ ) typical from hybrid bright giants. Given the paucity of empirical constraints on $v_{\mathrm{w}}$, we follow Drake \& Linsky (1986) and assume $v_{\mathrm{w}}=0.75 v_{\star}^{\text {esc }}$, where $v_{\star}^{\text {esc }}$ is the photospheric escape velocity. This relation is representative of the mean solar wind velocity and gives $v_{\mathrm{w}}=215 \mathrm{~km} \mathrm{~s}^{-1}$ for Pollux.

Drake \& Linsky (1986) showed that all the winds in their sample of coronal giants will be optically thin at $5 \mathrm{GHz}$. Following their arguments, we expect Pollux's wind to be optically thin at our 3 observed radio frequencies. We can derive an upper limit to the mass-loss rate by assuming the emission at 9 and $21.2 \mathrm{GHz}$ is entirely from other sources and not from the wind. Then, by extrapolating the power law fit to the flux densities at these frequencies (i.e., using $S_{v} \propto v^{1.5}$ ), we can obtain an effective flux density value at $3.0 \mathrm{GHz}$. The difference between our $3 \mathrm{GHz}$ upper limit for the flux density and this effective flux density is the upper limit to the flux density contribution from the wind and equates to $\sim 8 \mu \mathrm{Jy}$. We calculate the mass loss rate from a spherically symmetric, isothermal optically thin wind to be

$$
\begin{aligned}
\dot{M}_{\text {ion }}= & 1.3 \times 10^{-11} M_{\odot} \mathrm{yr}^{-1}\left(\frac{v_{\mathrm{w}}}{215 \mathrm{~km} \mathrm{~s}^{-1}}\right)\left(\frac{d}{10.36 \mathrm{pc}}\right) \\
& \times\left(\frac{T_{\mathrm{e}}}{10^{6.23} \mathrm{~K}}\right)^{0.22}\left(\frac{v}{3 \mathrm{GHz}}\right)^{0.03}\left(\frac{R_{\star}}{8.85 R_{\odot}}\right)^{0.5}\left(\frac{S_{\nu}}{\mu \mathrm{Jy}}\right)^{0.5}
\end{aligned}
$$

where $\dot{M}_{\text {ion }}$ is the ionized mass loss rate and $S_{v}$ is the maximum excess flux density at $3 \mathrm{GHz}$. For coronal giants, the ionized mass loss rate will be equal to the total mass loss rate, since hydrogen and helium are fully ionized. In deriving Eq. (5) we have computed the free-free Gaunt factor, $g_{\mathrm{ff}}$, assuming an effective power-law approximation to the appropriate hydrogen helium mix $[\mathrm{A}(\mathrm{He})=0.1]$ for coronal ionization conditions between $5 \times 10^{5}<T_{\mathrm{e}}(\mathrm{K})<10^{7}$ and VLA frequencies, i.e., $g_{\mathrm{ff}}=24.1 v^{-0.06} T_{\mathrm{e}}^{0.06}$. In addition, we have replaced the constant $v_{\mathrm{w}}$ with the beta power-law form $v_{\mathrm{r}}=v_{\mathrm{w}}\left(1-R_{\star} / r\right)^{\beta}$, where $r$ is radial distance. We adopt $r=1.2 R_{\star}$ as a limit to the integral calculation to be consistent with the chromospheric extensions inferred in Berio et al. (2011) and assume $\beta=1$ (Carpenter et al. 1995, 1999).

From Eq. (5) we can constrain the mass loss rate of Pollux to be $\dot{M}_{\text {ion }} \leq 3.7 \times 10^{-11} M_{\odot}$, which is about an order of magnitude smaller than previous constraints at radio wavelengths (Drake \& Linsky 1986). The semi-empirical mass loss relation from Schröder \& Cuntz (2005) predicts a mass-loss rate for Pollux that is almost identical to the value of our upper limit, while the scaling law from Holzer et al. (1983), which scales the solar mass loss rate by $\left(R_{\star} / R_{\odot}\right)^{2}\left(v_{\star}^{\text {esc }} / v_{\odot}^{\text {esc }}\right)^{-3}$, predicts a value that is a factor of two smaller. The theoretical model for mass-loss rates of cool stars by Cranmer \& Saar (2011) predicts $2.3 \times 10^{-12} \leq$ $\dot{M}_{\text {ion }} \leq 9.2 \times 10^{-12} M_{\odot}$, assuming $v \sin i=1.7 \mathrm{~km} \mathrm{~s}^{-1}$ (S. R. Cranmer 2016, priv. comm.). Our upper limit is therefore in agreement with existing predictions for the mass-loss rate of Pollux. The mass loss presumably occurs in magnetically open coronal regions, which account for only a portion of the total coronal free-free radio emission - the other portion coming from magnetically closed coronal regions. Since we have shown in Sect. 4.2.2 that the expected coronal free-free emission is about $0.5 \mu \mathrm{Jy}$, then it is probable that the actual mass-loss rate is considerably less than our derived upper limit.

\subsubsection{Exoplanetary emission}

The magnetized planets in our solar system produce electron cyclotron maser emission at very low frequencies (i.e., $v<$ $40 \mathrm{MHz}$ ) and it has long been suggested that this type of emission could potentially be detected from nearby magnetic exoplanets. Predictions for the strength of the exoplanet radio emission have been made whereby simple scaling laws known to operate in our solar system have been generalized for various stellar systems (e.g., Farrell et al. 1999; Stevens 2005). Pollux is known to have a planetary companion with a minimum mass of 2.9 $M_{\text {Jupiter }}$ and a semi-major axis of 1.6 AU (Reffert et al. 2006; Hatzes et al. 2006). If we optimistically assume that Pollux has a mass loss rate of $3.7 \times 10^{-11} M_{\odot} \mathrm{yr}^{-1}$ (see Sect. 4.2.4), then following Ignace et al. (2010) we could expect a contribution from exoplanetary radio emission of $\sim 25 \mu \mathrm{Jy}$ at $3 \mathrm{GHz}$, where we have assumed Jupiter-like properties for the rotation rate and radius of the planet and the emission to be isotropically beamed. However, since this type of radio emission is produced at the electron cyclotron frequency which is defined in Eq. (3) with $s=1$, the planetary magnetic field strength would need to be over $1000 \mathrm{G}$ in its polar region to be detectable at $3 \mathrm{GHz}$ and would need to be even larger to be observable at higher frequencies. Such large values are highly unlikely considering Jupiter has a value of only $14 \mathrm{G}$ and is the most magnetized planet in our solar system. We therefore do not expect to detect any exoplanetary emission in our data. Nevertheless, the larger mass loss rates of evolved stars in comparison to solar type stars could make them interesting candidates to search for exoplanetary emission at lower radio 
frequencies. For example, repeating the above calculation from Ignace et al. (2010) at $150 \mathrm{MHz}$ (i.e., a more realistic exoplanet emission frequency) predicts an exoplanetary radio flux density of $\sim 500 \mu \mathrm{Jy}$, which would be detectable with the Low Frequency Array (LOFAR).

\section{Conclusions}

The single coronal giant Pollux has been detected at 9 and 21.2 GHz with the VLA and tight upper limits for its flux density have been found at $3 \mathrm{GHz}$. The emission is thermal in nature and has disk-averaged brightness temperature values that are consistent with those of the quiet Sun. The origin of the emission is most likely from an optically thick upper chromosphere at 21.2 and $9 \mathrm{GHz}$. There may also be a small contribution from other mechanisms such as gyroresonance emission above active regions, an optically thin static (i.e., bound) corona and an optically thin stellar wind, particularly at the lower frequencies. We constrain the total mass loss rate of Pollux to be less than $3.7 \times 10^{-11} M_{\odot} \mathrm{yr}^{-1}$, which is about an order of magnitude smaller than previous constraints for coronal giants.

The frequency range covered in this paper is crucial for understanding stellar atmospheres because many different emission processes, like those discussed in this paper, occur over this range. The next generation of radio observatories such as the Square Kilometre Array (SKA) will have the sensitivity to better distinguish between theses competing processes. For example, Villadsen et al. (2014) argued that a detection of circular polarization would favour gyroresonance emission at these frequencies. This could be important for more active coronal giants than Pollux which presumably have more active regions on their observable disks. High $\mathrm{S} / \mathrm{N}$ detections at $3 \mathrm{GHz}$ and lower could trace emission from ionized stellar winds for coronal giants similar to Pollux. Indeed, detections of thermal continuum emission at 1.5 and $3 \mathrm{GHz}$ from non-cornal giants have already been used to constrain their partially ionized winds (O'Gorman et al. 2012). Furthermore, observations of nearby coronal giants at lower frequencies than those presented here could potentially be also used to search for exoplanetary radio emission. The detections presented in this paper are the first detections of radio emission from a single (i.e., non-binary) coronal giant. Pollux has the largest angular diameter of all coronal giants, and so the quiescent stellar disk dominated emission from other nearby single coronal giants will presumably be more difficult to detect using existing radio interferometers. The increased sensitivity of the SKA will enable radio surveys of nearby coronal giants to be carried out and will help elucidate the properties of coronal giant atmospheres as these stars evolve.

Acknowledgements. The data presented in this paper were obtained with the Very Large Array (VLA) which is an instrument of the National Radio Astronomy Observatory (NRAO). The NRAO is a facility of the National Science Foundation operated under cooperative agreement by Associated Universities,
Inc. This project was funded by ERC consolidator grant 614264. E.O.G. also acknowledges support from the Irish Research Council.

\section{References}

Athay, R. G. 1976, The solar chromosphere and corona: Quiet Sun, Astrophys. Space Sci. Lib., 53

Aurière, M., Wade, G. A., Konstantinova-Antova, R., et al. 2009, A\&A, 504, 231 Ayres, T. R. 1979, ApJ, 228, 509

Ayres, T. R., Fleming, T. A., \& Schmitt, J. H. M. M. 1991, ApJ, 376, L45

Ayres, T. R., Brown, A., Harper, G. M., et al. 1997, ApJ, 491, 876

Ayres, T. R., Simon, T., Stern, R. A., et al. 1998, ApJ, 496, 428

Ayres, T. R., Brown, A., Harper, G. M., et al. 2003, ApJ, 583, 963

Benz, A. O. 2009, Radio Emission of the Quiet Sun, Landolt Börnstein, Group VI, Astronomy and Astrophysics, Vol. 4B

Berio, P., Merle, T., Thévenin, F., et al. 2011, A\&A, 535, A59

Carpenter, K. G., Robinson, R. D., \& Judge, P. G. 1995, ApJ, 444, 424

Carpenter, K. G., Robinson, R. D., Harper, G. M., et al. 1999, ApJ, 521, 382

Cassinelli, J. P., \& Hartmann, L. 1977, ApJ, 212, 488

Condon, J. J., Cotton, W. D., Greisen, E. W., et al. 1998, AJ, 115, 1693

Cranmer, S. R., \& Saar, S. H. 2011, ApJ, 741, 54

Drake, S. A., \& Linsky, J. L. 1986, AJ, 91, 602

Drake, S. A., Simon, T., \& Brown, A. 1993, ApJ, 406, 247

Dupree, A. K., Lobel, A., Young, P. R., et al. 2005, ApJ, 622, 629

Farrell, W. M., Desch, M. D., \& Zarka, P. 1999, J. Geophys. Res., 104, 14025

Gray, D. F. 2014, ApJ, 796, 88

Gray, R. O., Corbally, C. J., Garrison, R. F., McFadden, M. T., \& Robinson, P. E. 2003, AJ, 126, 2048

Haisch, B., Schmitt, J. H. M. M., \& Rosso, C. 1991, ApJ, 383, L15

Harper, G. M., O'Riain, N., \& Ayres, T. R. 2013, MNRAS, 428, 2064

Hatzes, A. P., Cochran, W. D., Endl, M., et al. 2006, A\&A, 457, 335

Hatzes, A. P., Zechmeister, M., Matthews, J., et al. 2012, A\&A, 543, A98

Högbom, J. A. 1974, A\&AS, 15, 417

Holzer, T. E., Fla, T., \& Leer, E. 1983, ApJ, 275, 808

Huensch, M., Schmitt, J. H. M. M., Schroeder, K.-P., \& Reimers, D. 1996, A\&A, 310,801

Ignace, R., Giroux, M. L., \& Luttermoser, D. G. 2010, MNRAS, 402, 2609

Judge, P. G., Solomon, S. C., \& Ayres, T. R. 2003, ApJ, 593, 534

Lee, J., McClymont, A. N., Mikić, Z., White, S. M., \& Kundu, M. R. 1998, ApJ, 501,853

Linsky, J. L., \& Gary, D. E. 1983, ApJ, 274, 776

Liseau, R., Montesinos, B., Olofsson, G., et al. 2013, A\&A, 549, L7

Maggio, A., Vaiana, G. S., Haisch, B. M., et al. 1990, ApJ, 348, 253

McMullin, J. P., Waters, B., Schiebel, D., Young, W., \& Golap, K. 2007, in

Astronomical Data Analysis Software and Systems XVI, eds. R. A. Shaw, F. Hill, \& D. J. Bell, ASP Conf. Ser., 376, 127

Nordgren, T. E., Sudol, J. J., \& Mozurkewich, D. 2001, AJ, 122, 2707

O'Gorman, E., Harper, G. M., Brown, A., Drake, S., \& Richards, A. M. S. 2012, AJ, 144, 36

Parker, E. N. 1970, ARA\&A, 8, 1

Reffert, S., Quirrenbach, A., Mitchell, D. S., et al. 2006, ApJ, 652, 661

Sanz-Forcada, J., Micela, G., Ribas, I., et al. 2011, A\&A, 532, A6

Schröder, K.-P., \& Cuntz, M. 2005, ApJ, 630, L73

Sim, S. A. 2001, MNRAS, 326, 821

Spitzer, L. 1978, Physical processes in the interstellar medium (New York: Wiley-Interscience)

Stevens, I. R. 2005, MNRAS, 356, 1053

Takeda, Y., Sato, B., \& Murata, D. 2008, PASJ, 60, 781

van Leeuwen, F. 2007, A\&A, 474, 653

Villadsen, J., Hallinan, G., Bourke, S., Güdel, M., \& Rupen, M. 2014, ApJ, 788, 112

Wendker, H. J. 1995, A\&AS, 109, 177

White, S. M. 2004, New Astron. Rev., 48, 1319

White, S. M., \& Kundu, M. R. 1997, Sol. Phys., 174, 31

Wolff, S., \& Simon, T. 1997, PASP, 109, 759 